Annuaire suisse de politique de développement

$21 \mid 2002$

Agriculture suisse et mondialisation

\title{
9. Aide humanitaire
}

Xavier Tschumi Canosa

\section{OpenEdition}

\section{Journals}

Édition électronique

URL : http://journals.openedition.org/aspd/950

DOI : 10.4000/aspd.950

ISSN : 1663-9669

\section{Éditeur}

Institut de hautes études internationales et du développement

\section{Édition imprimée}

Date de publication : 1 avril 2002

Pagination : 299-307

ISSN : 1660-5934

\section{Référence électronique}

Xavier Tschumi Canosa, «9. Aide humanitaire », Annuaire suisse de politique de développement [En ligne], 21 | 2002, mis en ligne le 07 septembre 2012, consulté le 07 septembre 2020. URL : http:// journals.openedition.org/aspd/950; DOI : https://doi.org/10.4000/aspd.950 


\section{AIDE HUMANITAIRE*}

L

E CONSEIL FÉDÉRAL a adopté le nouveau "Message concernant la continuation de l'aide humanitaire internationale de la Confédération" le 14 novembre 2001. Le crédit-cadre proposé dans ce message s'élève à 1,5 milliard de francs et inclut pour la première fois la contribution fédérale au budget siège du CICR. L'Assemblée fédérale se prononcera sur ce crédit dans la première moitié de 2002.

Les versements d'aide humanitaire au titre de l'APD se sont élevés, en 2000, à 337 millions de francs, en baisse globalement de $15 \%$ par rapport à l'année précédente. Cette baisse concerne les versements bilatéraux, tout particulièrement en faveur de l'Europe du Sud-Est, alors que les versements multilatéraux ont augmenté entre 1999 et 2000.

Le 28 septembre 2001, le Conseil fédéral a décidé de modifier le nom du Corps suisse d'aide en cas de catastrophe (ASC), qui est devenu le Corps suisse d'aide humanitaire (CSA).

\subsection{RAPPEL DU CADRE INSTITUTIONNEL ET FINANCIER}

C'est la DDC qui détient la compétence de l'aide humanitaire, selon la Loi fédérale sur la coopération au développement et l'aide humanitaire (du 19 mars 1976) et son ordonnance d'application (du 12 décembre 1977). Au sein de la DDC, c'est le secteur Aide humanitaire et ASC (Corps suisse d'aide en cas de catastrophe) qui prend la responsabilité des actions.

Les ressources financières nécessaires au déroulement de ces actions sont prévues dans le cadre de crédits de programmes pluriannuels, proposés par le Conseil fédéral au Parlement sous la forme d'un message (voir ci-dessous, point «Nouveau Message concernant la continuation de l'aide humanitaire»). Les montants effectifs alloués à l'aide humanitaire sont discutés et déterminés chaque année lors des débats sur le budget fédéral, et sont puisés alors dans le crédit-cadre.

Si la DDC est compétente en matière d'aide humanitaire au niveau de la Confédération, d'autres départements et services fédéraux peuvent aussi en financer certains aspects, notamment en ce qui concerne l'assistance aux réfugiés. De leur côté, les cantons et communes suisses opèrent aussi des versements au titre de l'aide humanitaire, de même que les ONG suisses, sur leurs ressources propres.

Les domaines d'engagement de l'aide humanitaire suisse sont la prévention, le sauvetage, la survie et la reconstruction.

Dans son aide humanitaire, la DDC recourt à quatre instruments distincts : l'envoi de personnel (de l'ASC), les contributions financières, l'aide alimentaire et l'envoi de matériel. La DDC opère par des interventions directes (effectuées en

* Par Xavier Tschumi, licencié en économie et diplômé de l’IUED, Genève. 
grande majorité par l'ASC, mais aussi par les ambassades suisses ou les bureaux de coordination de la DDC) et des contributions aux organisations internationales et aux ONG suisses. Dans ses contributions, la DDC recourt aux quatre instruments susmentionnés.

\section{$\square$ Nouveau «Message concernant la continuation de l'aide humanitaire»}

Le crédit-cadre actuel, portant de 1997 à 2001 au moins et d'un montant de 1050 millions de francs, sera remplacé par un nouveau, après les sessions de printemps et d'été 2002 du Parlement. Le message du Conseil fédéral en ce sens, adopté le 14 novembre 2001, propose d'allouer 1500 millions à l'aide humanitaire internationale, sur une durée d'au moins quatre ans. Trois raisons essentielles expliquent cette importante augmentation du crédit-cadre:

- pour la première fois, ce crédit-cadre intègre la contribution au budget siège du Comité international de la Croix-Rouge (CICR) ${ }^{1}$, alors que cette dernière faisait auparavant l'objet d'un message et d'un crédit-cadre spécifiques (280 millions de francs) ;

- le Conseil fédéral veut réaliser son objectif de hisser la part de l'APD dans le PNB suisse à $0,4 \%$;

- le Conseil fédéral veut augmenter l'engagement humanitaire de la Confédération.

Le Message du Conseil fédéral concernant la continuation de l'aide humanitaire internationale de la Confédération ${ }^{2}$ débute par une analyse de l'état du monde vu par les acteurs humanitaires à l'aube du troisième millénaire ainsi que des défis auxquels ces acteurs seront confrontés. Il se poursuit par une description synthétique de l'aide humanitaire de la Confédération, notamment en tant qu'instrument de la politique extérieure de la Suisse et dans le cadre des relations existant entre cet instrument et les autres instruments de politique extérieure, en particulier les politiques de sécurité, de développement et de migration ou le commerce extérieur.

Le message comprend ensuite une présentation détaillée du crédit-cadre luimême, ainsi que sa ventilation entre les différents moyens.

Tableau 30: Ventilation du crédit-cadre selon les moyens de l'aide humanitaire internationale, pour une durée de 4 ans au moins (en millions de francs)

\begin{tabular}{lcc}
\hline Moyens de l'aide humantaire & $\begin{array}{c}\text { Nouveau } \\
\text { crédit-cadre }\end{array}$ & $\begin{array}{c}\text { Précédent } \\
\text { crédit-cadre }\end{array}$ \\
\hline Actions directes & 180 & 110 \\
\hline CICR & 420 & 91 \\
\hline Contributions financières à des actions humanitaires & 550 & 489 \\
\hline Aide alimentaire en produits laitiers d'origine suisse & 140 & 140 \\
\hline Aide alimentaire en céréales & 100 & 110 \\
\hline Réserve extraordinaire pour situation de détresse & 110 & 110 \\
\hline
\end{tabular}

Source: Conseil fédéral, Message concernant la continuation de l'aide humanitaire internationale de la Confédération, non encore coté officiellement (état décembre 2001).

1. Les contributions aux actions du CICR sur le terrain étaient par contre toujours incluses dans le crédit-cadre de l'aide humanitaire (montant proposé par le Conseil fédéral dans son nouveau message: 140 millions de francs).

2. Ce message n'a pas encore été coté officiellement par la Chancellerie fédérale (décembre 2001). 
En annexe de ce message sont enfin présentées les priorités géographiques et sectorielles de l'aide humanitaire de la Confédération qui ont été financées par le précédent crédit-cadre.

\subsection{VERSEMENTS D'AIDE HUMANITAIRE EN 2000}

En 2000, les versements d'aide humanitaire de la Suisse au titre de l'APD se sont élevés à près de 337 millions de francs, contre plus de 398 millions en 1999 (61,4 millions de moins, soit environ 15\%). Ce sont les versements bilatéraux qui ont diminué $(63,6$ millions de moins, soit une diminution d'environ $17 \%)$, alors que les versements multilatéraux ont augmenté $(2,4$ millions de plus, soit une augmentation d'environ $11 \%$ ).

Tableau 31: Versements d'APD de la Suisse au titre de l'aide humanitaire en 2000, par émetteur et par instrument (en millions de francs)

\begin{tabular}{lcccr}
\hline & $\begin{array}{c}\text { Aide } \\
\text { humanitaire }\end{array}$ & $\begin{array}{c}\text { Aide } \\
\text { alimentaire }\end{array}$ & $\begin{array}{c}\text { Assistance } \\
\text { aux réfugiés }\end{array}$ & Total \\
\hline Versements bilatéraux & $\mathbf{2 0 3 . 1}$ & $\mathbf{3 0 . 2}$ & $\mathbf{7 9 . 5}$ & $\mathbf{3 1 2 . 8}$ \\
\hline DDC & 198.1 & 30.2 & 0.0 & 228,3 \\
\hline Office fédéral des réfugiés (ODR) & 0.0 & 0.0 & 79.5 & 79,5 \\
\hline Cantons et communes & 5.0 & 0.0 & 0.0 & 5,0 \\
\hline Versements multilatéraux & $\mathbf{2 4 . 2}$ & $\mathbf{0 . 0}$ & $\mathbf{0 . 0}$ & $\mathbf{2 4 . 2}$ \\
\hline DDC & 24.2 & 0.0 & 0.0 & 24,2 \\
\hline Total & $\mathbf{2 2 7 . 3}$ & $\mathbf{3 0 . 2}$ & $\mathbf{7 9 . 5}$ & $\mathbf{3 3 7 . 0}$ \\
\hline
\end{tabular}

Source: DDC, service statistique, septembre 2001

\section{Emetteurs et instruments de l'aide humanitaire}

En 2000, la DDC a fourni près des trois quarts de l'aide humanitaire suisse, dont la totalité de l'aide alimentaire et de l'aide multilatérale.

Le quart restant a été fourni essentiellement par l'ODR au titre de l'assistance aux réfugiés $(23,5 \%)$ et pour une très petite partie par les cantons et communes suisses, au titre de l'aide humanitaire (voir plus bas).

Aide humanitaire fournie par le Corps suisse d'aide en cas de catastrophe (ASC)

Dans le cadre de l'aide humanitaire, la DDC compte avec son Corps suisse d'aide en cas de catastrophe (ASC), lequel est intervenu directement dans 43 pays en 2000. En outre, il a participé au travail de cinq organisations internationales (PAM, HCR, OIM, OCHA et CICR) dans huit pays supplémentaires (pour la répartition géographique des interventions de l'ASC, voir plus bas).

L'ASC a déboursé 63,9 millions de francs au titre de l'APD, dont 32,4 millions sur les fonds de la DDC, et le reste sur ceux de l'ODR au titre de l'assistance aux réfugiés (voir plus bas).

\section{Contributions bilatérales d'aide humanitaire à des organisations tierces}

La DDC a fourni des contributions pour des programmes spécifiques d'aide humanitaire à différentes organisations, en particulier à celles du Mouvement international de la Croix-Rouge. Le CICR a reçu 95,2 millions de francs en 2000, dont 67,2 millions au titre de la contribution générale de la Confédération 
à cette organisation. La Croix-Rouge suisse (CRS) a reçu 5,6 millions de francs et la Fédération internationale des sociétés de la Croix-Rouge et du CroissantRouge (FICR) 2,4 millions.

En outre, la DDC a fourni des contributions pour des programmes spécifiques d'aide humanitaire à plusieurs organisations internationales, dont le Programme alimentaire mondial (PAM) pour un montant de 10,6 millions de francs, le Haut-Commissariat des Nations Unies pour les réfugiés (HCR) pour un montant de 9 millions, le Fonds des Nations Unies pour l'enfance (UNICEF) pour un montant de 4,6 millions et le Bureau de coordination des affaires humanitaires des Nations Unies (OCHA) pour un montant de 4,1 millions.

Enfin, la DDC a soutenu le travail de diverses ONG suisses actives dans le domaine de l'aide humanitaire, notamment Caritas et Terre des Hommes (Lausanne) pour un montant respectif de 3,5 et 3,2 millions de francs, l'Entraide protestante suisse (EPER) pour un montant de 1,4 million, Medair pour un montant de 1,3 million, Vétérinaires sans frontières pour un montant de 1,2 million et Médecins sans frontières Suisse pour un montant de 1 million.

\section{Aide alimentaire}

L'aide alimentaire a été entièrement financée par la DDC, à hauteur de 30,2 millions de francs en 2000. Elle consiste essentiellement en produits laitiers suisses (plus de 2500 tonnes) et en céréales (près de 22'000 tonnes). Les deux tiers de cette aide alimentaire (en valeur) sont passés par des organisations internationales (le PAM presque exclusivement) et le tiers restant par des ONG suisses (notamment Caritas).

\section{Versements multilatéraux au titre de l'aide humanitaire}

En 2000, la DDC a versé 24,2 millions de francs au titre de l'aide humanitaire multilatérale. Les principales organisations bénéficiaires furent le HCR (13 millions de francs), l'Office de secours et de travaux des Nations Unies pour les réfugiés de Palestine au Proche-Orient (UNRWA, 9,4 millions) et l'Organisation internationale pour les migrations (OIM, 1 million).

\section{Assistance aux réfugiés}

L'assistance aux réfugiés peut être assimilée à de l'aide humanitaire et certaines dépenses y relatives entrer dans l'agrégat d'APD. Dans l'assistance aux réfugiés entrent en ligne de compte l'aide en Suisse, l'aide au retour des réfugiés dans leurs pays d'origine ainsi que l'aide sur place. En ce qui concerne les dépenses effectuées dans les pays du CAD en faveur des réfugiés, le calcul des montants pouvant être comptabilisés dans l'APD est controversé et varie considérablement entre ces pays. La Suisse se tient, quant à elle, à des critères plutôt restrictifs pour ce calcul: les dépenses effectives d'APD liées à l'assistance aux réfugiés se sont élevées, en 2000, à 133,3 millions de francs ${ }^{3}$. L'ODR a versé 79,5 millions de francs à ce titre, dont 54,7 millions au titre de l'aide au retour (le Kosovo étant le principal bénéficiaire de ce montant, avec 45,2 millions). La DDC gère une partie des montants versés par l'ODR et contribue également, sur ses propres ressources, aux programmes d'assistance aux réfugiés.

3. Ce montant a été calculé par la DDC et seul apparaît dans le tableau 31 le montant des contributions d'APD fournies par l'ODR au titre de l'assistance aux réfugiés. Le reste est inclus dans l'aide humanitaire. 


\section{Aide humanitaire des cantons et communes suisses}

En 2000, les cantons et communes suisses ont fourni une aide humanitaire à hauteur de 5 millions de francs. Cette aide a bénéficié à de nombreuses ONG suisses, dont la CRS (0,8 million de francs) et la Chaîne du bonheur ( 0,3 million).

\section{$\square$ Répartition géographique de l'aide humanitaire en 2000}

Outre les versements bilatéraux d'aide humanitaire qui ne sont pas géographiquement ventilés, c'est l'Afrique qui est la principale bénéficiaire de cette aide en 2000, suivie de l'Europe, de l'Asie, du Moyen-Orient et de l'Amérique latine. Par rapport à 1999, les versements bilatéraux d'aide humanitaire en faveur de l'Europe ont chuté de plus de moitié, demeurant cependant bien supérieurs aux montants versés à cette région dans les années 1997 et 1998.

ASTM 2001, p. 350.

Tableaux 32: Versements bilatéraux d'aide humanitaire en 2000, par continent et par pays (en millions de francs)

\begin{tabular}{|c|c|c|}
\hline & Total & dont de la DDC \\
\hline Afrique & 60.6 & 59.5 \\
\hline Soudan & 9.2 & 9.2 \\
\hline Mozambique & 6.6 & 6.4 \\
\hline Congo (Rép. dém.) & 6.2 & 6.1 \\
\hline Angola & 5.9 & 5.9 \\
\hline Burundi & 4.9 & 4.9 \\
\hline Ethiopie & 4.7 & 4.7 \\
\hline Sierra Leone & 4.5 & 4.5 \\
\hline Europe & 45.1 & 24.4 \\
\hline République fédérale de Yougoslavie (RFY) & 15.3 & 10.8 \\
\hline dont Serbie seule & 11.4 & 8,1 \\
\hline Moldavie & 1.9 & 1.9 \\
\hline Croatie & 1.5 & 0.3 \\
\hline ex-République yougoslave de Macédoine (ERYM) & 1.3 & 1.3 \\
\hline Albanie & 1.3 & 1.3 \\
\hline Asie & 24.5 & 23.9 \\
\hline Afghanistan & 6.6 & 6.6 \\
\hline Corée (Rép. dém.) & 3.1 & 3.1 \\
\hline Géorgie & 2.7 & 2.7 \\
\hline Inde & 1.8 & 1.8 \\
\hline Sri Lanka & 1.7 & 1.7 \\
\hline Tadjikistan & 1.4 & 1.4 \\
\hline Moyen-Orient et Afrique du Nord & 13.6 & 12.8 \\
\hline Irak & 5.9 & 5.9 \\
\hline Iran & 2.4 & 2.4 \\
\hline Turquie & 1.5 & 1.5 \\
\hline Algérie & 1.4 & 1.4 \\
\hline Amérique latine & 13.4 & 12.9 \\
\hline Colombie & 4.5 & 4.5 \\
\hline Honduras & 2.7 & 2.7 \\
\hline Venezuela & 1.0 & 0.9 \\
\hline Suisse & 4.9 & 4.9 \\
\hline Non ventilé géographiquement & 150.7 & 89.9 \\
\hline Total & 312.8 & 228.3 \\
\hline
\end{tabular}

Source: DDC, Service statistique, septembre 2001. 


\section{Distribution géographique de l'aide humanitaire fournie par l'ASC}

L'ASC est intervenu directement et indirectement dans 51 pays en 2000, dont 44 appartiennent à la partie I de la liste des pays bénéficiaires de l'aide établie par le CAD (voir en fin d'Annuaire). Seules les interventions dans ces derniers pays peuvent être comptabilisées dans l'agrégat d'APD.

En 2000, les dépenses de l'ASC au titre de l'aide humanitaire ont bénéficié majoritairement à l'Europe du Sud-Est (près de 48 millions de francs ${ }^{4}$, dont 30,7 millions financés par l'ODR au titre de l'assistance aux réfugiés).

L'ASC est aussi intervenu en Afrique (5,3 millions de francs), notamment en Angola ( 2 millions) et en République démocratique du Congo (1,4 million). En faveur de l'Amérique latine, l'ASC a déboursé 4,3 millions, surtout en Amérique centrale (3,4 millions dont 1,6 million pour le Honduras). L'Asie a bénéficié de l'aide de l'ASC à hauteur de 1,6 million, notamment la République démocratique de Corée ( 0,9 million). Certaines dépenses en Suisse de l'ASC ont également été comptabilisées comme APD (pour un montant de 4,3 millions).

Enfin, l'ASC est intervenu au titre de l'aide alimentaire pour un montant de 0,7 million, en Moldavie essentiellement.

Distribution géographique de l'aide humanitaire des cantons et des communes

L'aide humanitaire des cantons et communes suisses a bénéficié à plus de 50 pays, en particulier en Europe (montant total de 1,2 million de francs, essentiellement en faveur de la RFY) et en Afrique (1,1 million). En outre, la Turquie a absorbé 0,8 million de francs de cette aide.

\section{Distribution géographique de l'aide alimentaire (DDC)}

L'aide alimentaire de la DDC a été répartie sur plus de 40 pays en 2000, dont les principaux sont le Soudan (2,8 millions de francs), l'Irak (2,5 millions) et l'Afghanistan ( 2 millions). L'Afrique est la principale bénéficiaire de cette aide (11,4 millions), suivie de l'Asie (4,5 millions) et du Moyen-Orient (3,8 millions). 7,7 millions de francs ont également été déboursés au titre de l'aide alimentaire sans spécification géographique des pays destinataires.

Distribution géographique de l'assistance aux réfugiés (ODR)

L'assistance au réfugiés financée par l'ODR a essentiellement bénéficié à l'Europe du Sud-Est. Dans le seul programme d'aide au retour, l'ODR a versé 45,2 millions de francs à la DDC, qui agit comme agence de mise en œuvre du programme au Kosovo (aide structurelle).

4. Ce chiffre est supérieur à celui qui est indiqué dans le tableau 32 (45,1 millions), du fait de deux versements négatifs concernant l'assistance aux réfugiés (ODR): 11,8 millions en provenance de la Bosnie-Herzégovine et 11,9 millions en provenance du Kosovo. 


\subsection{ENGAGEMENT DE LA SUISSE EN EUROPE DU SUD-EST ${ }^{5}$}

En termes d'aide humanitaire, l'engagement de la Suisse dans les pays d'Europe du Sud-Est considérés en développement a été considérablement moindre en 2000 qu'en 1999, année de la guerre au Kosovo. Les programmes d'aide humanitaire dans cette région avaient été, en 1999, les plus importants jamais réalisés par la Suisse.

띠 ASTM 2001, p. 351.

Au Kosovo en particulier, l'aide d'urgence de la Suisse fait progressivement place, en 2001, à des programmes de coopération au développement à long terme visant à reconstituer les bases économiques, sociales et politiques de cette région. En dépit de cette tendance, la Suisse a tout de même continué de participer aux opérations de déminage dans cette province. En 2001, la DDC a versé 1,5 million de francs (puisés sur les fonds de l'ODR au titre de l'aide au retour) en faveur du programme de déminage coordonné par l'ONU et mis en œuvre par Emercom (ministère russe en charge des questions humanitaires).

Dans un nouveau message ${ }^{6}$ concernant la participation de la Suisse à la Force multinationale de maintien de la paix au Kosovo (KFOR), le Conseil fédéral propose de prolonger l'engagement du contingent de la Swiss Company (Swisscoy) jusqu'à la fin de l'année 2003. Dès le mois d'octobre 2002 cependant, les membres de la Swisscoy seront équipés d'une arme personnelle et ne dépendront plus, pour leur propre protection, du contingent autrichien de la KFOR; cela fait suite à l'approbation par le peuple suisse, lors de la votation du 10 juin 2001, de l'envoi de soldats suisses armés à l'étranger. Ce message a été débattu aux Chambres fédérales dans leur session d'hiver 2001, mais des divergences sont demeurées au sein du Conseil national.

La Commission européenne et la Banque mondiale ont coorganisé une conférence internationale à Bruxelles, le 29 juin 2001, pour évaluer les besoins de la RFY après le transfèrement de Slobodan Milosevic à La Haye. La Suisse y a participé en prévoyant d'allouer 40 millions de francs à ce nouveau pays de concentration en 2001 et 95 millions sur trois ans. La RFY a rejoint, le 8 mai 2001, le groupe de vote conduit par la Suisse dans les institutions de Bretton Woods et sera soutenue par la Suisse dans son intégration au sein de ces institutions, tant que ce pays progressera sur la voie des réformes démocratiques. Pour la seule année 2001, les promesses d'aide issues de la conférence se sont élevées à 1,28 milliard de dollars, pour des programmes humanitaires mais, surtout, pour des programmes de coopération au développement. Cette somme s'adresse uniquement à la Serbie et au Monténégro, et exclut le Kosovo, placé sous administration de l'ONU et ayant fait l'objet d'une précédente conférence internationale.

5. Les pays usuellement comptés sous cette dénomination sont l'Albanie, la Bosnie-Herzégovine, la Croatie, l'ex-République yougoslave de Macédoine, la République fédérale de Yougoslavie (comprenant la Serbie, le Monténégro et le Kosovo), ainsi que la Bulgarie et la Roumanie. La Bulgarie et la Roumanie sont des pays en transition, contrairement aux autres pays qui sont, eux, en développement.

6. Conseil fédéral, Message concernant l'arrêté fédéral sur la participation de la Suisse à la Force multinationale de maintien de la paix au Kosovo (KFOR) du 12 septembre 2001, Feuille fédérale, pp. 5771-5782 (message 01.055). 


\section{Engagement actuel de la Suisse en RFY}

Aide humanitaire (DDC) - accueil et logement des réfugiés, reconstruction d'écoles, d'hôpitaux et d'installations de chauffage, réparation des dégâts écologiques occasionnés par la guerre. Budget $2001:$ 7,5 millions de DEM.

Coopération technique (DDC) - réforme des institutions étatiques et de l'administration publique, soutien aux communes et à l'éducation, promotion des PME, intégration et protection des minorités ethniques. Budget 2001 : 12 millions de DEM.

Coopération financière (Seco) - reconstruction des infrastructures électriques, développement du secteur privé, promotion du commerce et des investissements. Budget $2001: 27$ millions de DEM.

Pacte de stabilité - promotion des médias, renforcement de la démocratie locale et de la coopération régionale, éducation et formation professionnelle, appui à la cohésion sociale.

Source: DDC et Seco, Conférence internationale des donateurs de la République fédérale de Yougoslavie. La Suisse veut poursuivre son engagement, communiqué de presse, 25.6.01.

\subsection{ELÉMENTS D'IMPORTANCE TOUCHANT AU THÈME DE L'AIDE HUMANITAIRE EN 2001}

La conférence annuelle de l'Aide humanitaire et du Corps suisse d'aide en cas de catastrophe (ASC) s'est déroulée le 30 mars 2001 à Berne et a porté sur le thème "Suisse humanitaire - ONU humanitaire: convergence». Le commissaire général de l'UNRWA et le haut-commissaire adjoint du HCR étaient présents à cette conférence, leurs agences respectives étant les principales bénéficiaires de l'aide humanitaire multilatérale de la Suisse (voir ci-dessus point «Emetteurs et instruments de l'aide humanitaire»).

La DDC a également publié une brochure ${ }^{7}$ sur sa coopération avec l'ONU, dans le domaine de la coopération au développement et dans celui de l'aide humanitaire. Cette brochure passe en revue l'engagement de la DDC dans les principaux fonds et programmes de l'ONU ainsi que leurs principales thématiques actuelles.

Le 28 septembre 2001, le Conseil fédéral a décidé de donner un autre nom à l'ASC, «Corps suisse d'aide humanitaire» (CSA), jugeant la précédente dénomination trop restrictive.

En outre, le Conseil fédéral a réglé pour la première fois de manière exhaustive, par une nouvelle ordonnance adoptée le 24 octobre 2001, l'aide en cas de catastrophe fournie à l'étranger par la Confédération et les cantons frontaliers. Ce texte optimise les compétences des différents départements et services fédéraux, cantonaux et communaux.

7. DDC, La DDC et l'ONU. Coopération au développement. Aide humanitaire, 2001. Cette brochure est disponible sur le site Internet de la DDC, <www.ddc.admin.ch>. 


\section{SOURCES}

Conseil fédéral, Message concernant la continuation de l'aide humanitaire internationale de la Confédération, non encore coté officiellement (état décembre 2001).

DDC, «Kosovo. Début de l'aide à long terme», Newsletter, $\mathrm{n}^{\circ} 3$, juin 2001.

-, La Suisse soutient les opérations de déminage au Kosovo, communiqué de presse, 15.6.01.

DFAE, 1500 millions proposés pour l'aide humanitaire de la Confédération, communiqué de presse, 14.11.01.

L'AGEFI, «La République fédérale de Yougoslavie engrange les promesses financières après l'extradition de Milosevic», 2.7.01.

Neue Zürcher Zeitung, «Milliarden-Aufbauhilfe für Jugoslawien. Zusicherung der internationalen Geberkonferenz», 30.6.01.

\section{SITES INTERNET}

Comité international de la Croix-Rouge: <www.icrc.org>.

Corps suisse d'aide humanitaire: <www.skh.admin.ch>.

DDC : <www.ddc.admin.ch>. 